\title{
The Multimedia Reference Model: A Frame- work Facilitating the Creation of Multi-User, Multimedia Applications
}

\author{
Stephan Abramowski, Karin Klabunde, Ursula Konrads, Karl Neunast, \\ Hermann Tjabben \\ Philips Research Laboratories \\ P.O. Box 1980, D-52021 Aachen, Germany \\ Tel.: +492416003 535, Fax: +492416003518 \\ E-mail: neunast@pfa.philips.de
}

\begin{abstract}
This paper focuses on the application control functionality required for future interactive multi-user, multimedia applications. Accordingly a new service control reference model is described which is part of a more general Multimedia Reference Model. This service control reference model introduces application building blocks that facilitate application creation and management in all kinds of multi-user, multi-point and multi-channel environments in interactive video and other multimedia broadband networks. The Multimedia Reference Model is derived by applying the conceptual modelling technique standardized for Intelligent Networks by ITU-T. Based on the service control reference model, a distributed functional architecture is developed which contains - among others - the functionality for application control, session management, and security handling. The functional design of the architecture enables it to be mapped in various ways onto the physical network components, for example, in a set-top box/ video server configuration. The new Multimedia Reference Model is applied to an interactive multi-user video games environment.
\end{abstract}

\section{INTRODUCTION AND OVERVIEW}

The advent of digital compressed video and fibre optic communication has opened up new avenues for advanced multimedia applications and services ${ }^{1}$. Much progress has been made concerning the standardization of video compression techniques (MPEG1 and 2), the development of new equipment such as video servers, digital set-top boxes etc. and the deployment of the

1. Throughout this paper the terms 'application' and 'service' are deliberately used as synonyms. 
enabling network infrastructure (fibre/coax, FTTC). However, little effort has been spent up till now on the important issue of application control.

Some first aspects of application control, particularly as regards session control are dealt with in the ITU-T.120 series of recommendations on videoconferencing and multi-point communication [1]. Further elaboration of the session control concept can be found in the work carried out in the Bellcore Touring Machine system [2] and Expanse [3] projects.

Further standardization work for multimedia network and service control is being done in the ATM Forum and in the ITU-T study groups in the framework of the Broadband ISDN User Part (BISUP) [4] and ISCP ([5], [6]). However, Capability Set 1 (CS-1) of BISUP will be limited to setting up simple, single-connection calls; only CS-3 will add multimedia and broadcast connections (however, no date of completion of work has yet been specified [7]). Advanced control concepts are discussed in [8] and [9], also some preliminary discussion on service control is taking place in the DAVIC (Digital Audio-Video Council) project [10].

All the aforementioned activities do not yet meet the requirements of flexible multimedia application creation and management. Those requirements are:

- The need for a reference model. The complexity of networked multimedia systems makes a reference architecture indispensable, that treats the different views to the system (end user, application designer, provider, operator, etc.) and the relations between them. This also models the relationship between the application and the system infrastructure.

- The need for abstraction. Application designer need not to know all details of the underlying networked system. The provided system capabilities must be offered through adequate abstractions at application programming interfaces (APIs). The suitability of an API is given if no application designer sees the need to use lower level system interfaces, e.g. operating system calls for communication purposes.

- The need for re-use. The required APIs can be provided through re-usable building blocks offering well-tried solutions for the commonalities of networked multimedia applications, such as setting up and managing a multimedia conference.

The modelling techniques of the Intelligent Network Conceptual Model (INCM) by ITU-T (see [11]) proved to be a powerful means for the definition and communication of interfaces and protocols (as shown f.i. in the PHIDES [12] system for service creation and the Open Switching platform [13]), and turned out to be extensible for multimedia systems in general.

This paper further extends the scope of the Intelligent Network (IN) by describing a service control reference model which is part of a more general Multimedia Reference Model and can deal with all kinds of multi-user, multi-point and multi-channel environments in interactive video and other multimedia broadband networks. In section 2 the Multimedia Reference Model is derived by applying the conceptual modelling technique developed in IN. To maintain the INCM concept to construct the applications from application independent building blocks, we introduced Application Building Block (ABB) objects. The ABBs form an application construction set that facilitates application creation and management. Based on the service control reference model, a distributed functional architecture is developed which contains - among others - the functionality for application control, session management, and security handling. The functional design of the architecture enables it to be mapped in various ways onto the physical network components, for example, in a set-top box/video server configuration. This also includes a more flexible mapping of the cooperation protocols at the distributed functional 
plane onto signalling protocols at the physical plane. In section 3 the new architecture is applied to an interactive multi-user video games environment.

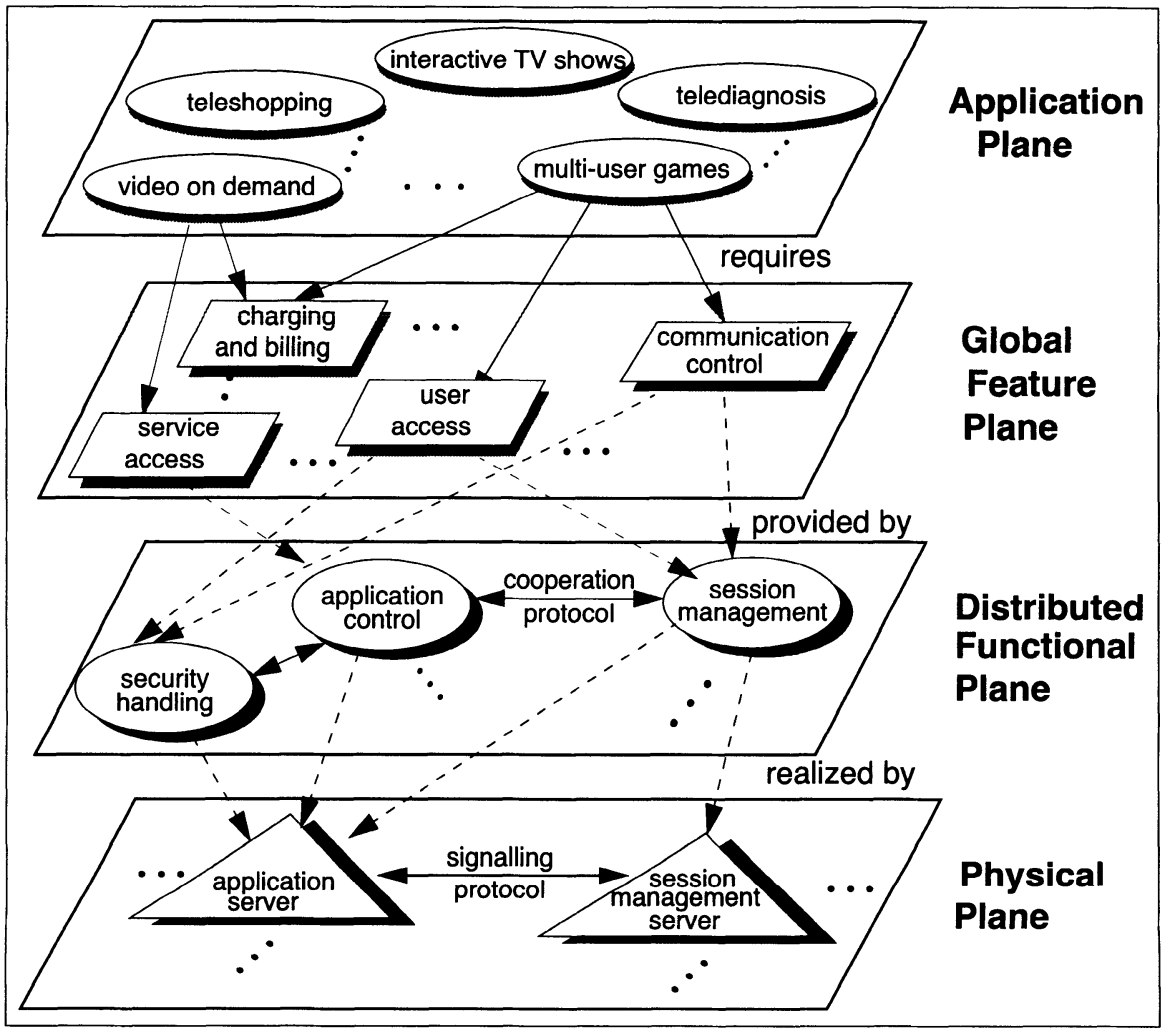

Figure 1 Multimedia Reference Model

\section{MULTIMEDIA REFERENCE MODEL}

For complex systems, such as networked multimedia systems, an overall conceptual framework is strongly needed. The knowledge and the interests of all concerned parties are too different to meet their needs with a single view to the system. Different views have to be offered to the end user, the application designer, provider, and the operator as well as to the network operator and the component manufacturer. Figure 1 shows the Multimedia Reference Model that we developed from the INCM, based on our experiences with the realization of IN concepts in form of the service creation environment PHIDES [12] and of the Open Switching platform [13].

The Application Plane describes the end user's view to the offered applications. Examples for such applications are telediagnosis, teleshopping, interactive TV shows, video on demand, or multi-user games. We replaced the INCM's term 'Service Plane' by 'Application Plane' be- 
cause 'service' is a generic notion, (e.g. each of the seven layers of the OSI reference model offers its 'services' to the next higher layer).

The Global Feature Plane defines the application building blocks (ABB) of the networked, interactive, multi-user, multimedia applications described on the Application Plane. Those application building blocks are combined to construct the service logic of an application. The Service Control Reference Model is part of the Global Feature Plane. It is described in section 2.1 with special emphasis on the communication control ABB. In Figure 1 the features charging and billing, service access, user access, and communication control are shown exemplary. These application independent features take care of the commonalities among the different applications. Communication control refers to the logical ability to bring two or more parties together and to allow them to participate in a multimedia session.

We maintained the INCM idea to construct the applications from application independent building blocks, but dropped the CS-1 Service Independent Building Blocks (SIBs) and their chaining, which proved to be impractical.

The Distributed Functional Plane describes the functional entities and cooperation protocols between them, defining a functional architecture. Application control, security handling and session management are examples of important system functions to be provided by a distributed multimedia system. Other functions are e.g. network management, subscription and subscriber management and information access function. The functional architecture is defined such that it can provide the functionality needed for the application building blocks at the global feature plane.

The Physical Plane describes the possible distribution scenarios that can reach from centralized realization of single-vendor solutions to the maximum distribution where every functional entity of the Distributed Functional Plane is realized as a physical component by an independent organisation.

\subsection{Global Feature Plane}

The Service Control Reference Model of the Global Feature Plane offers a simplified view to the networked multimedia system, suitable for an application designer. Within the application logic many aspects of multimedia communication are hidden by abstractions which we call Application Building Block (ABB). These Application Building Blocks are software objects which offer the needed logical view via their operations. They are used by the application objects that are written by the application designer. We offer seven Application Building Blocks on the Global Feature Plane of our Multimedia Reference Model. These seven ABBs are shown on the right side of Figure 2, which outlines their use in a multi-user game application. This figure only indicates the 'use' relation between an application instance, i.e. multi-user game, and the ABBs. It does not give a complete specification of a service logic and of the timely order of the invocations.

In the following, we describe as an example the communication control object class which is a very important one. Before we present our solution - the communication control $A B B$ - we give an overview of the state-of-the-art in communication control abstractions.

\subsubsection{State-of-the-Art in Communication Control Abstractions}

From the many activities in the field of multimedia communication control abstractions we selected those that influenced our approach. These are ITU-T's T.120 series for multi-point com- 


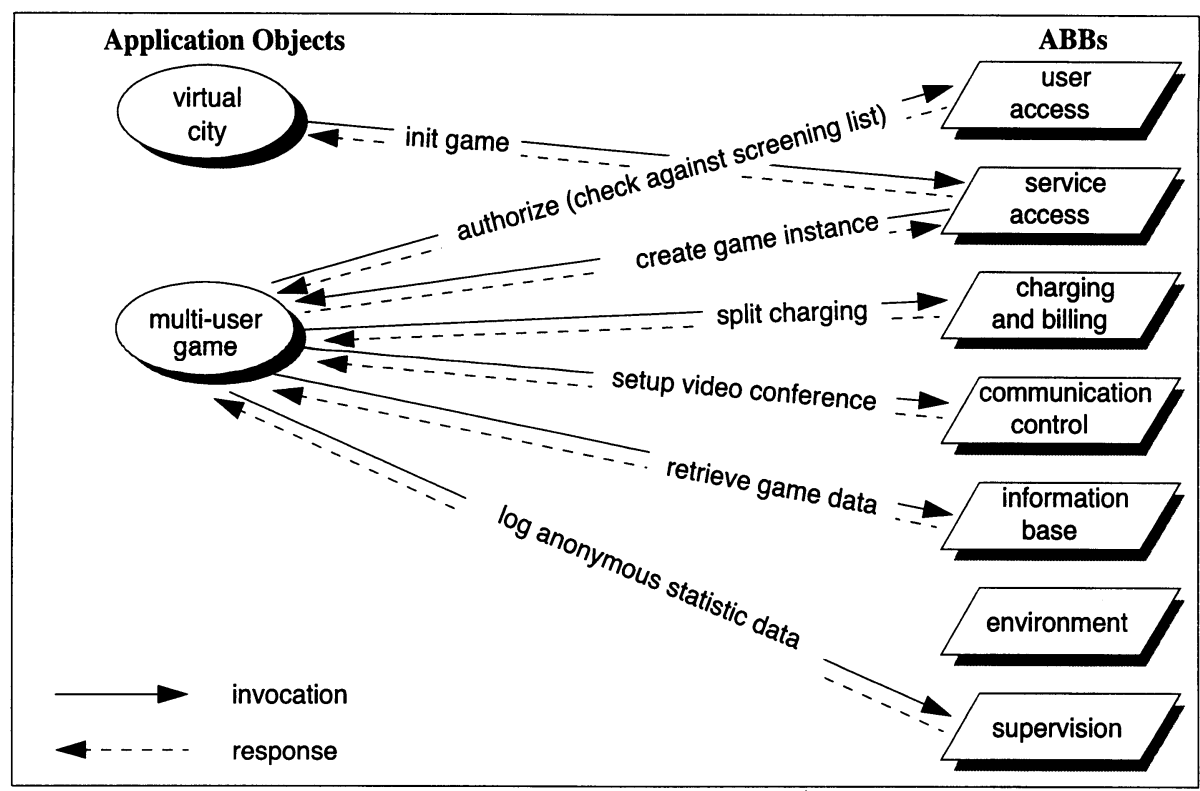

Figure 2 Sample interaction relation between the application objects and the ABBs

munication, Bellcore's Touring Machine and Twente's Service Elements. We will briefly describe them here.

\section{Multi-point Communication Services And Generic Conference Control}

During the last few years the ITU-T developed a set of data protocols for multimedia communications that permit group working between multiple - geographically dispersed - parties, see Figure 3. These protocols are published in the T.120 series [1]. Although these protocols were originally intended mainly for desktop videoconferencing it is now recognized that they are useful for a much wider range of application areas within the field of multi-point, multimedia communications.

There are two main characteristics of the T.120 standards that make them quite attractive. First, the T.120-protocols are applicable to a wide range of different networks profiles, e.g. PSTN, ISDN or PSDN. Second, within the T.120 system model certain standardized protocol entities are already offered that make use of the services defined in the lowest multi-point layer - the 'Multipoint Communication Services' and optionally of the services defined in the package 'Generic Conference Control'. Among these protocol entities are packages for 'Still Imaging' and 'Audio Visual Control'. However, it is also possible for users to develop their own nonstandard application protocol entities that reside on top of MCS and GCC. End-user applications can then be developed that only use services that are defined in these dedicated protocol entities.

The MCS protocol defines primitives for connection, domain, channel and token management. GCC uses the MCS protocol to offer more enhanced services for conference establishment and 


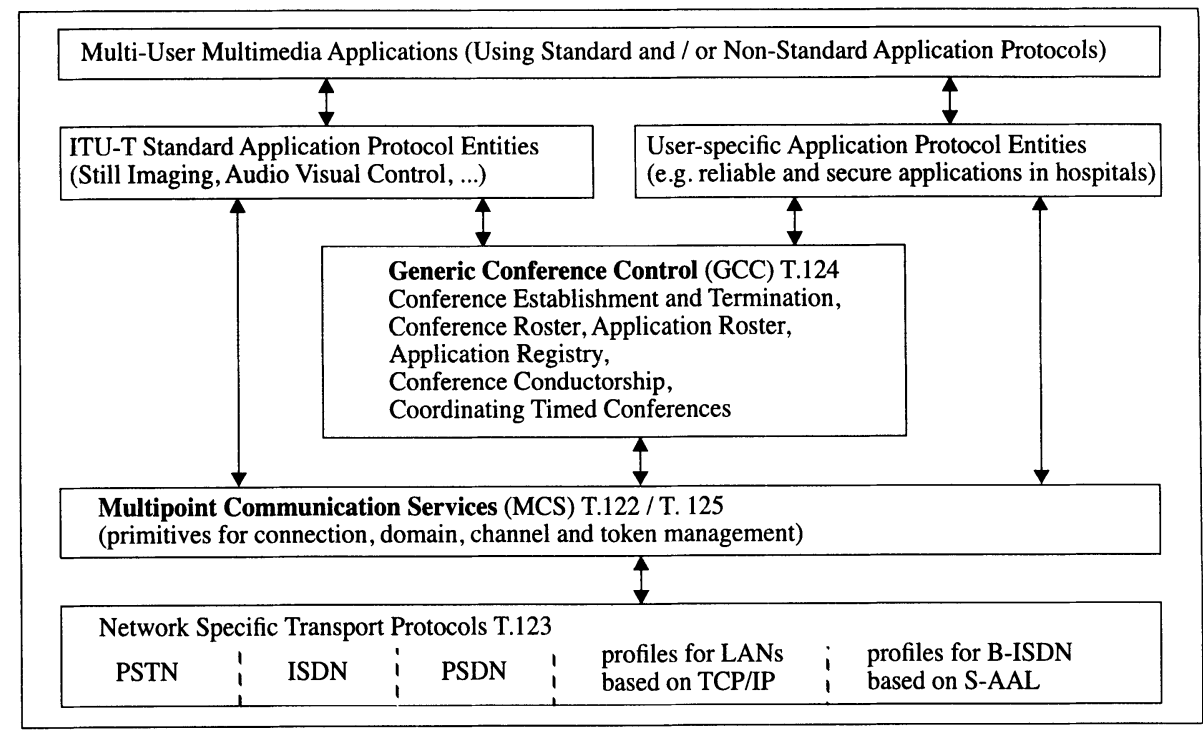

Figure 3 ITU-T's T.120 protocol stack

termination, conference and application roster, application registry, conference conductorship and coordinating timed conferences.

Because of the standardized interfaces and of the successive abstraction layers the T.120 protocols fit well into our concept of a multimedia reference architecture. The T.120 series is interesting with respect to the network transparency it offers. To reach a high degree of flexibility concerning the underlying networks we will consider to realize application independent building blocks on top of the MCS resp. GCC layer. However, the distribution of functionality and the identification of the various network components is not yet well addressed within the T.120 series. For the issue of an explicit representation of session topology we will now look at Bellcore's Touring Machine.

\section{Bellcore's Touring Machine System}

The idea behind Bellcore's Touring Machine ${ }^{1}$ system is to provide a common platform which facilitates the development and operation of complex multimedia applications [2]. The applications can be developed using an Application Programming Interface (API) which hides the details of the underlying physical networks. The Touring Machine API offers capabilities to establish, and modify multiple concurrent multi-user multimedia communication sessions.

The transport topology of a communication session can be specified using a set of logical abstractions. Figure 4 shows the logical view on a three-party multimedia session. The abstractions used by the Touring Machine are: connectors, endpoints and ports.

A connector is an abstraction of a communication bridge and can have several types: data, audio and video. The termination points of a connector are logical ports which are called endpoints. Endpoints are characterized by the medium, the direction of flow and the connected client who provides respectively receives transport. Endpoints are mapped onto ports which

1. Touring Machine is a trademark of Bellcore. 


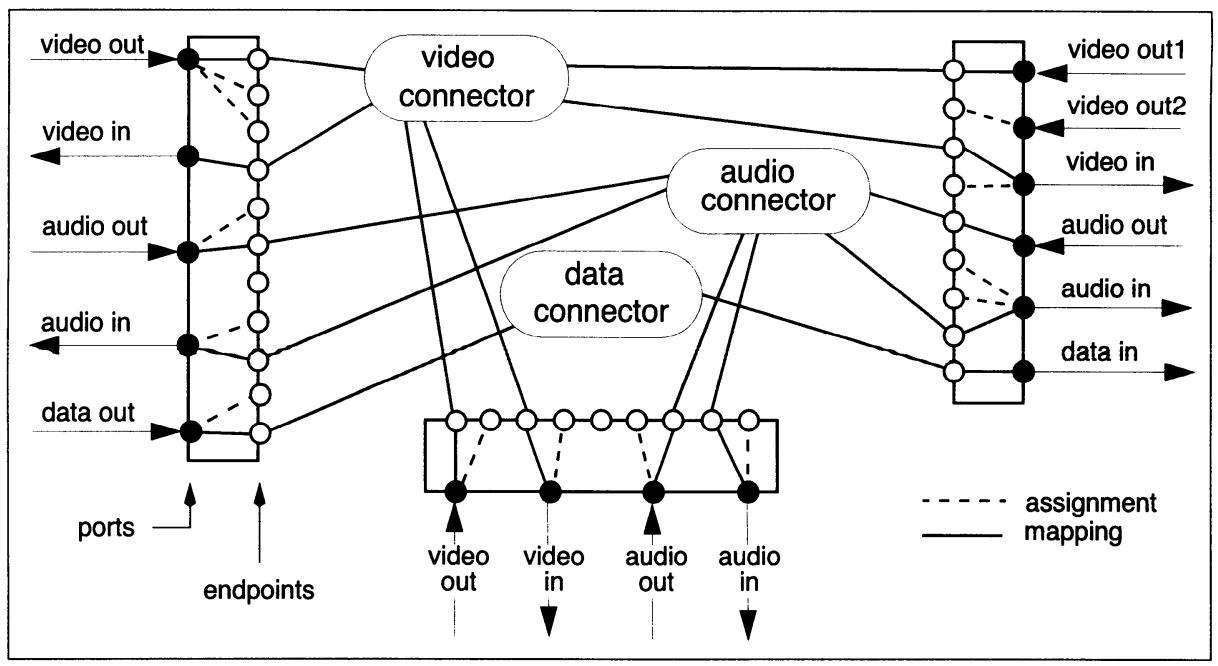

Figure 4 Touring Machine: three-party multimedia session

are network access channels to which a client can attach its data/audio/video equipment. Ports are characterized by medium and direction of flow. A client specifies how its endpoints shall be mapped to the ports. This assignment can be changed dynamically during a session thus allowing to share devices between concurrently running sessions.

The three-party multimedia session shown has three connectors, the video and audio connector have a multi-point connection whereas the data connector shows a point-to-point connection. All three parties have an in/out-connection to the video connector, i.e. they can all send and receive video. The same applies for the audio connector. The data connector is only attached to two parties, one party is sending data ('data out') and the other party is receiving data ('data in').

The logical view on the topology of a session as proposed by Touring is quite attractive although we think that possibly more abstractions are needed: the development of applications without knowledge about ports is desirable. Additionally, the collection of privileges, i.e. in and out, and the access mechanisms controlling them should be further developed. Furthermore, there is no representation of synchronization relations between different media.

\section{Twente's Abstract Service Elements}

Heijenk, Hou, and Niemegeers from the University of Twente described a set of abstract service elements [14] that enables to characterize networked multimedia applications. The Twente approach uses the abstractions of the Touring Machine API, but the connectors are called media. Additionally, an explicit synchronization relation between those media was introduced. Major aim of this work is to come to an agreement about a complete, explicit and precise description of the functional behaviour of a communication system supporting multi-user multimedia applications.

We consider this work as highly valuable - perhaps for a slightly different reason as it was originally intended by the authors - because the offered service description takes place on a very abstract level. The communication system is regarded as a whole, i.e. lower-layer details 


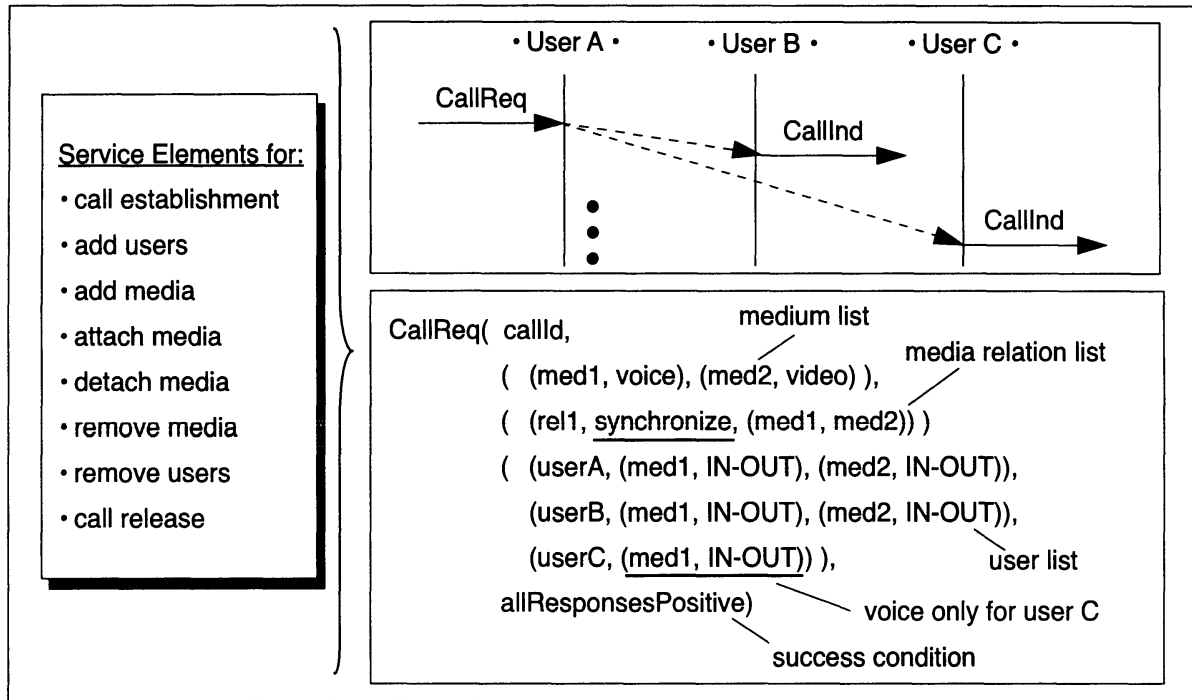

Figure 5 Call establishment with Twente's abstract service elements

remain hidden and no assumption is made about the distribution of the functionality in the network. It is explicitly stated in the article that only the required capabilities of a communication system are investigated but that nothing is said about the realization and how interactions between service primitives have to be resolved. Application developers benefit from such an approach because they can use the set of abstract service elements as building blocks for multiuser, multimedia applications without having in-depth knowledge of the underlying communication technologies.

We sketch the possible use of the proposed service elements which are listed in Figure 5. For each service element there exist several primitives (e.g. CallReq, CallInd). We do not go into details of the negotiation process, e.g. a request service is received as an indication message by the addressed parties and usually has to be responded. Parameters of the call establishment primitive CallReq are the list of media to be used in the call, the assumed synchronization relations between these media and the list of called users together with the media aimed at in that connection. The success condition prescribes when a call request is accepted. The call identifier is used to later add users or media to an existing call.

\subsubsection{Communication Control ABB}

The application designers need an abstract view on the capabilities of the multimedia communications system. Therefore details of the distribution of the functionality of the various components in a network and of their physical realization should be kept hidden as much as possible. Nevertheless it is important to visualize the constructs for the creation of multi-user multimedia applications that are offered by the network in a way that stimulates the process of creating new networked multimedia examples. In the following, we describe the session model and the API of the communication control $\mathrm{ABB}$, our means to offer the required abstract view. 


\section{Session Model}

The session model of the communication control ABB offers an abstract view to the session topology and to session management. This representation does not treat the state models for the different components of the session model. The state models introduce the detection points which offer the hooks for the event handling by the application.

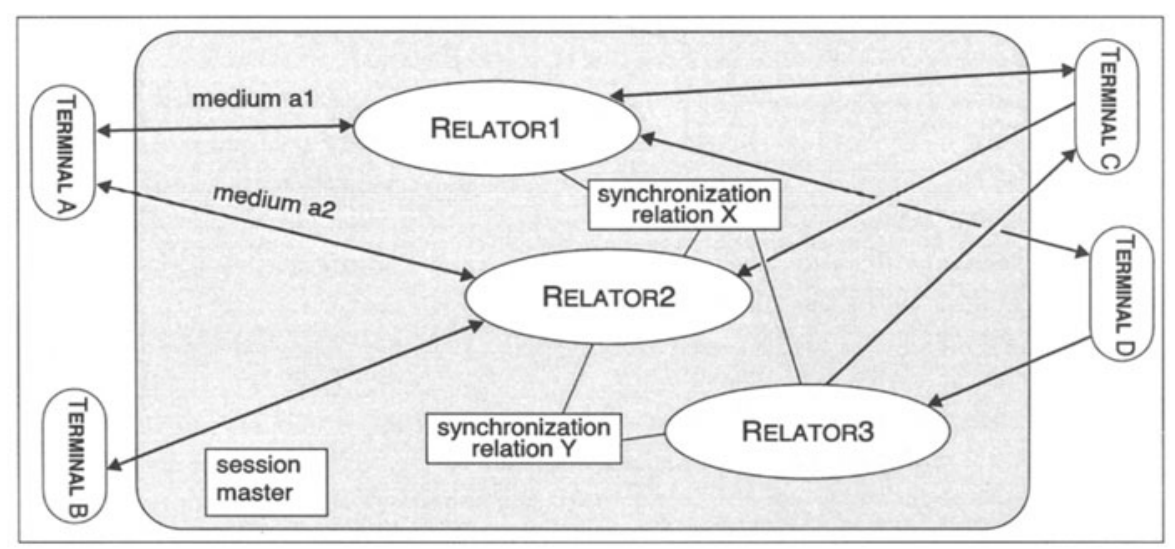

Figure 6 Topology of a four-party multimedia session

The following constructs are introduced by the session model: relator, medium, synchronization relation, session master.

The relator is a further development of the Touring Machine connector [2]. A relator supports sophisticated connect relations going far beyond the simple in or out of an audio/video bridge. Examples for those sophisticated connect relations are complex audio or video mixing or the support of the virtual city paradigm. The video mixing could combine input of a video camera (e.g. the head of a person) with application data (e.g. presentation of the body an artificial person) to produce a new actor of the application who is partially real and partially taken from a fantasy world. With the virtual city paradigm an application can diminish the volume of an input corresponding to a distance relevant in a virtual city application. This could be an audio conference of actors in a virtual pub where the audio input is diminished proportional to the actors distance to a virtual table. An example for the support of the virtual city paradigm is the product "The Parallel Universe" offered by the Virtual Universe Corporation.

The medium ${ }^{1}$ represents the communication path between a terminal and the corresponding relator, e.g. of type audio. We introduced the synchronization relation, as proposed by Heijenk et al. [14] but not further described, to explicitly arrange synchronism between media, e.g. to keep video and audio of a movie lip-synchronous.

The session master is responsible for the composition and control of a session (e.g. granting privileges), and for the charging arrangements, e.g. splitting of charges between participants of a session. The application itself can also be the session master, e.g. for a meet-me-conference of the babble box type.

1. A medium is comparable to the Twente attachment, while a relator is comparable to the Twente medium. 
Figure 6 shows the topology of a multimedia session with four terminals connected via three relators which are synchronized by two synchronization relations.

\section{Communication Control API}

The messages establishing the communication control API are:

- changeTopology which adds respectively removes a new construct to respectively from a session, i.e. a medium, a relator, a synchronization relation, or even creates a complete session.

- changePrivilege which supports a wide variety of communications privileges of the participants in an application. Besides the classic privileges 'read' (i.e. also 'listen' or 'watch') and write (i.e. also 'speak' or 'show'), sophisticated ones are provided like 'read but not copy'.

- changeQuality which is used for the modification of quality of service characteristics (such as bandwidth, delay, jitter, or synchronization). Additionally, the required confidence of communicated data is arranged with this operation.

- setDP which makes the explicit event handling for the multi-user, multimedia session possible. Detection points (DPs) can be activated for a collection of session events. Examples of possible session events are (session) created and partyAdded (see also Figure 10). If the corresponding event occurs, the DP fires and the communication control object informs the application objects. The basis of our DP mechanism are the ITU-T recommendations for IN [11].

- sessionEvent which informs the application objects about relevant session events. The relevance of a session event is determined by the application logic itself using the operation setDP.

\subsection{Distributed Functional Plane}

The service features of the Global Feature Plane have to be provided by functional entities (FEs) on the Distributed Functional Plane. These functional entities and their cooperation protocols construct the functional architecture of the multimedia system. Figure 7 shows the functional entities of the Distributed Functional Plane that provide the communication control ABB at the Global Feature Plane.

As it is indicated in Figure 7, the FEs security handling, subscription management, and several instances of session management at the Distributed Functional Plane provide communication control to the Global Feature Plane. In order to do so, these FEs cooperate with each other, and with other FEs (session management cooperates with transport network control, for instance). An example of typical cooperation protocols involved is given below (see Figure 10).

\subsection{Physical Plane}

For every physical component of a multimedia system the physical plane gives the functional entities that reside in the physical component. Figure 11 gives one possible mapping for a networked multi-user multimedia game system with set-top boxes, that are connected to a central 


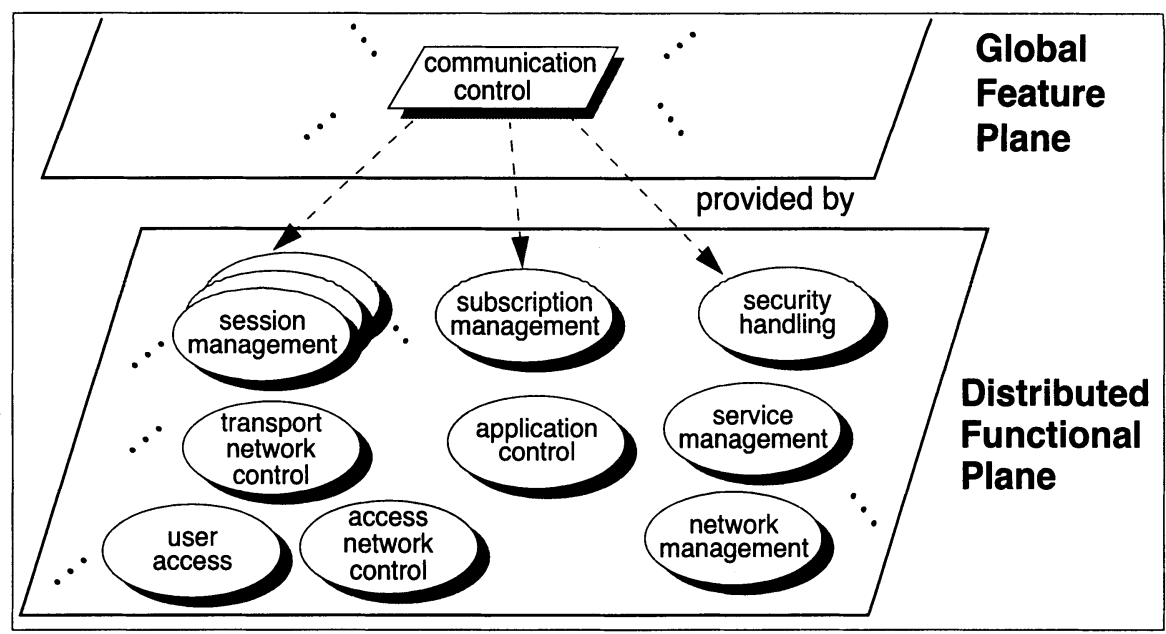

Figure 7 Mapping the system feature communication control onto functional entities

application server through a network. Service Creation and Management reside in their own physical components. The functional entities may be mapped and distributed in various ways into the physical components in the physical plane. It is the advantage of the $\mathrm{IN}$ conceptual model to provide a maximum of flexibility for such distribution scenarios.

\section{VIEWS ON AN INTERACTIVE, MULTI-USER GAME}

The four planes of the Multimedia Reference Model allow to present four different views to a multimedia system and its applications. In this section we illustrate this by a multi-user game example called Telepainter.

\subsection{The Application Plane View}

The Application Plane describes the end user's view to the offered application:

Telepainter is a networked multi-user game for two teams of players. In each round one player has to paint a term from one of six categories; the other players in his team have to guess the term from the painting as fast as possible. Meanwhile, the other team just watches how the active team is performing.

To offer a comfortable playing atmosphere, the players communicate via audio and video connections, in addition to the connections for the painting information and the control buttons.

Similar, non-electronic versions of this game idea are called Images, Pictionary, Montagsmaler etc.

\subsection{The Global Feature Plane View}

The ABB object classes of the Global Feature Plane form an application construction set that enables to easily create a new application by re-using these well-tried solutions for the commo- 


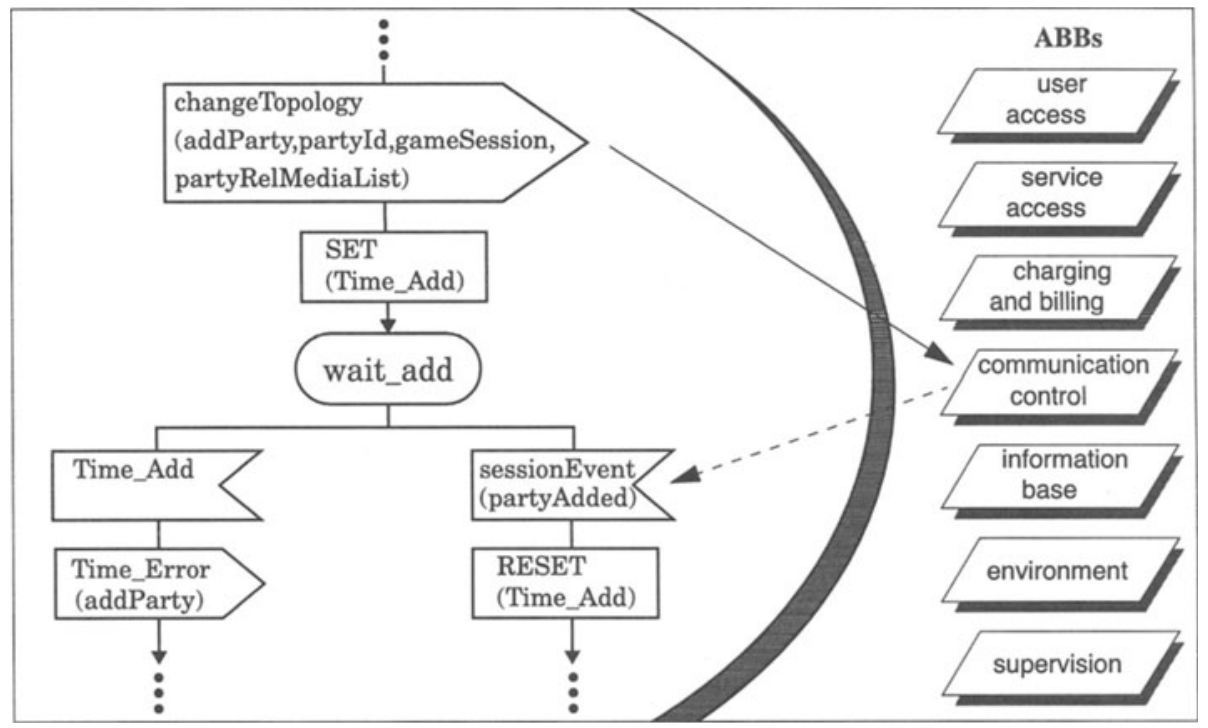

Figure 8 Sample of the Telepainter logic as SDL diagram using the ABB communication

nalities of networked multi-user, multimedia applications. The multi-user game object in Figure 2 represents the service control logic of the Telepainter game. There it is sketched how the application logic uses the Application Building Blocks (ABBs) to check the invited players, to setup the audio/video/data session, to arrange the charging among the players, to retrieve Telepainter terms, and to log some statistic data concerning the game.

In Figure 8 we outline how the application logic of the Telepainter game requests an operation of the communication control ABB and handles the resulting sessionEvent message. We use SDL ${ }^{1}$ for the specification of the dynamic model of OMT [15]. The use of OMT results in an object-oriented design. The ABB object classes, such as the communication control ABB, can be used in every object-oriented development environment. Figure 9 outlines its possible use in a $\mathrm{C}++$ environment. In this example the 
service logic of a multi-user game application uses the communication control ABB to add a player to the multi-user, multimedia session.

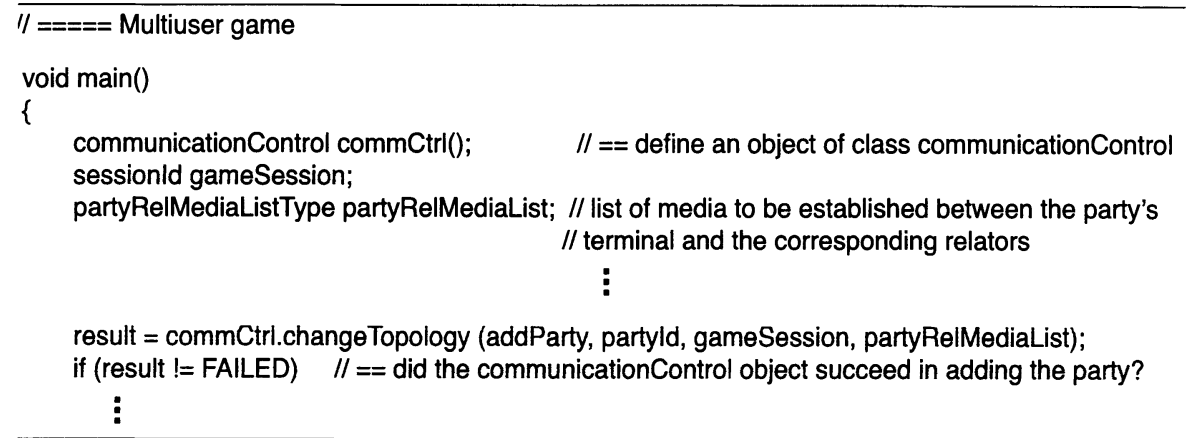

Figure 9 Sample use of the communication control object class in a $\mathrm{C}++$ syntax

\subsection{The Distributed Functional Plane View}

The Distributed Functional Plane describes the functional entities and cooperation protocols between them.

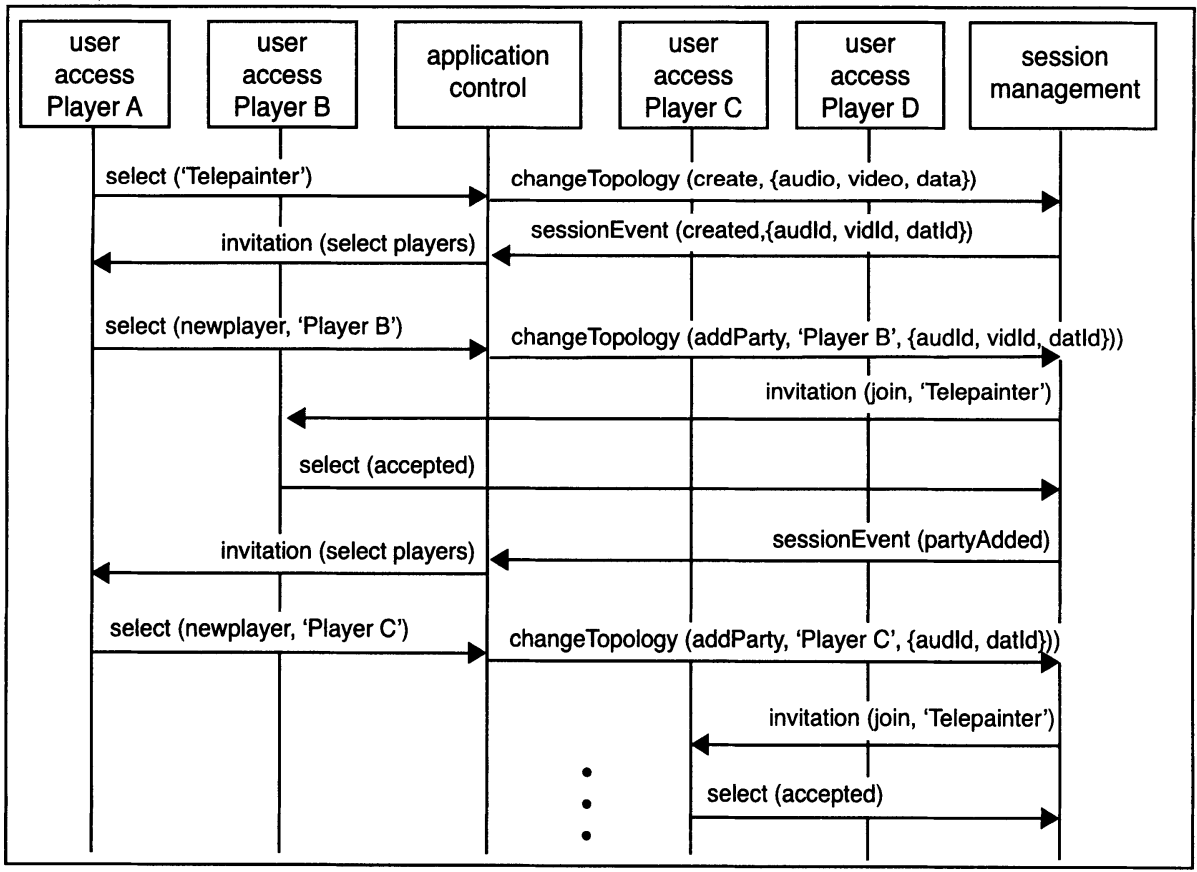

Figure 10 Sample message sequence for the start of a Telepainter playing round 
Figure 10 outlines how some of those functional entities cooperate to start a Telepainter playing round. The involved functional entities are application control function, session management function, and four instances of user access function. The observed cooperation 'changeTopology' at the Distributed Functional Plane directly realizes the 'changeTopology' instruction the service logic gave in Figure 2 at the Global Feature Plane. We intentionally chose the same names for the operations at the DFP and at the GFP in this case. But in principal, operations at the DFP and at the GFP are different, and in general there is no one-to-one mapping between them.

\subsection{The Physical Functional Plane View}

A possible mapping to a physical architecture for multi-user games is shown in Figure 11. The session management functionality resides in this mapping example together with application control in the application server. Furthermore, the network must provide audio and video connections and highly reactive control channels for user control via control buttons. The mapping between the cooperation protocols at the distributed functional plane and the signalling protocols at the physical plane is not shown. The control information may be transported either via common channel signaling (i.e. SS7) including client-server control via user-user signaling, or partly via separate control channels between clients and servers. Another possibility currently being explored (see [4]) is to use a signaling protocol based on the OSI application layer service in an ATM environment.

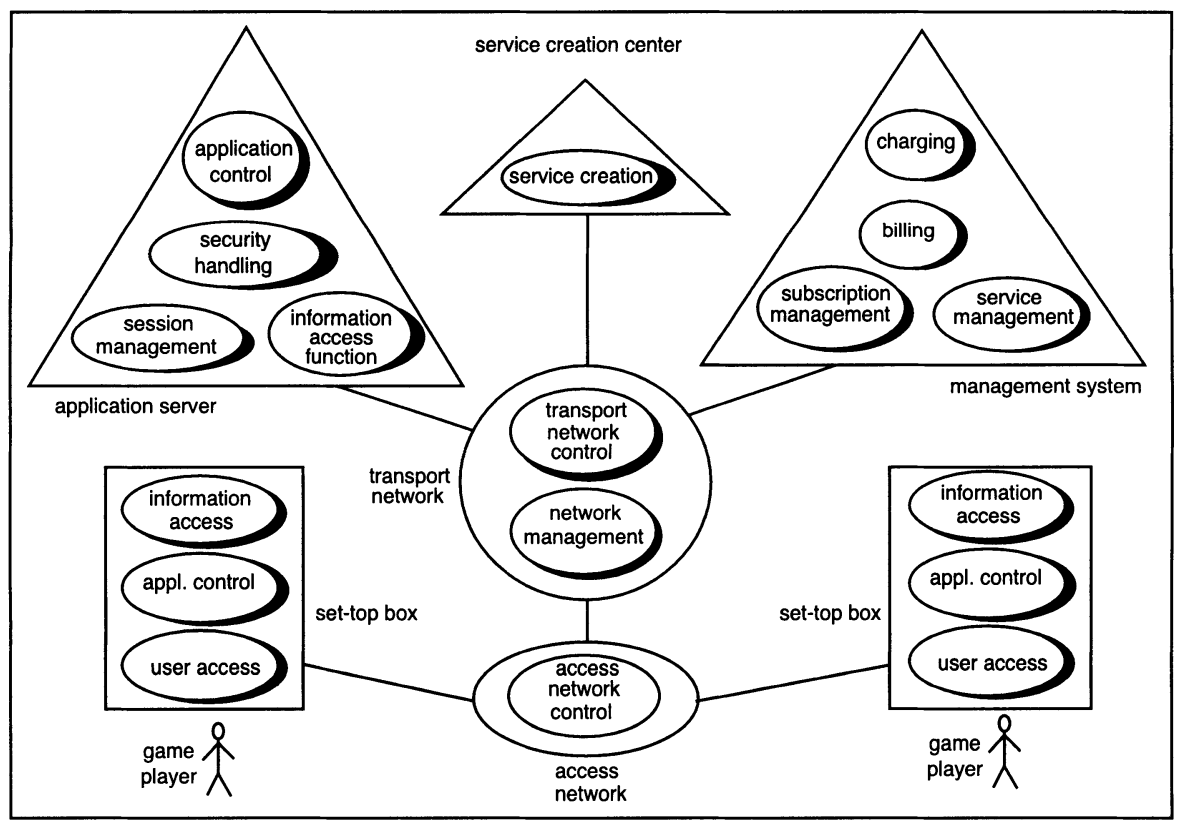

Figure 11 Possible mapping of functional entities to a physical architecture 


\section{CONCLUSIONS}

The 'Intelligent Network Conceptual Model' [11] turned out to be a valuable tool in the development of 'intelligent networks', by establishing an accepted terminology and providing a framework for architectural discussions. A similar approach, that we called Multimedia Reference Model, can be followed in the field of multimedia, where layered architectural considerations are still largely missing (ATM, B-ISDN, DAVIC, ..), and may turn out to be equally valuable there. We showed the usefulness of the approach by modelling application control aspects, especially session management aspects. We introduced Application Building Blocks as the re-incarnation of the INCM idea to construct applications from application independent building blocks. We replaced the CS-1 SIBs and their chaining by a collection of object-oriented ABBs with their operational interface. These ABB object classes meet the requirements of flexible multimedia application creation and management: The need for a reference model, the need for abstraction, the need for re-use.

We applied our approach to an interactive multi-user, multimedia game, presenting the four different views to the Telepainter game.

\section{REFERENCES}

[1] ITU-T, Study Group 8; T.120: Transmission Protocols for Multimedia Data; 20 March 1995

[2] M. Arango et al.; The Touring Machine system; Communications of the ACM, January 1993, Vol. 36, No. 1

[3] S. Minzer; A Signaling Protocol for Complex Multimedia Services; IEEE Journal on Selected Areas in Communications, December 1991

[4] B. Law; Signalling in the ATM network; BT Technol. J., Vol.12, No.3, July 1994

[5] ITU-TSS WG 11/2 - COM 11-R 10-E; ISCP Baseline Document; Version 3.1, Geneva, June 1993

[6] A. Paglialunga, Biocca, A., Siviero, M.; Signalling protocol for broadband ISDN; Proc. ICC' $92,322.3$

[7] P.J. Kühn, Pack, C.D., Skoog, R.A.; Common Channel Signaling Networks: Past, Present, Future; IEEE Journal on Selected Areas in Communications, Vol.12, No.3, April 1994

[8] T.F. La Porta, Veeraraghavan; Design and Implementation of a Distributed Call Processing Architecture; Proceedings of the IEEE International Conference on Communications, May 1-5, 1994

[9] T.F. La Porta, 5, M. et al.; B-ISDN: A Technological Discontinuity; IEEE Communications Magazine, October 1994

[10] N.N.; responses to DAVIC's CFP; DAVIC 5th Meeting, Tokyo, 4-7 December 1994, Parts 1-3

[11] CCITT; Q.1211 - Q.1218 Draft Recommendations; CCITT SG XI, March 1992

[12] S. Abramowski, M. Elixmann, H. Gappisch, U. Heister, U. Heuter, K. Klabunde; $A$ Service Creation Environment for Intelligent Networks; Proceedings of the 1992 Int. Zurich Seminar on Digital Communication, March 16 - 19, 1992, Zurich, Switzerland 
[13] M. Elixmann, B.L. de Greef, A.M.M. Lelkens, K.W. Neunast, H.Tjabben; Open Switching - Extending Control Architectures to Facilitate Applications; Proceedings of the ISS '95, Berlin, Germany, April 23 - 28, 1995

[14] G.J. Heijenk, X. Hou, I.G. Niemegeers; Communication Systems Supporting Multimedia Multi-user Applications; IEEE Network, January/February 1994

[15] J. Rumbaugh, M. Blaha, W. Premerlani, F. Eddy, W. Lorensen; Object Modeling and Design; Prentice-Hall, 1991

\section{BIOGRAPHY}

Karl W. Neunast studied Electrical Engineering at the Technical University of Aachen, Germany, where he graduated in Technical Informatics and received his doctor's degree for his thesis about security mechanisms of operating systems. Since 1990 Dr. Neunast has been with the Philips Research Laboratories in Aachen working on call control and service switch-ing in intelligent networks, especially for ITU-T IN and for ECMA CSTA. Actually Dr. Ne-unast is engaged in the architectural design of networked multimedia systems, in the protocol specification of their functional entities, and in the development of a creation methodology for networked, multi-user, multimedia applications.

Stephan Abramowski studied Computer Science at the Technical University of Karlsruhe, Germany, where he graduated in 1985 and received his doctor's degree in 1989 in the field of computer graphics and computational geometry. Since 1990 he has been with Philips Research Laboratories in Aachen, working in projects on service and network architectures, especially service creation in Intelligent Networks for value-added voice services and session management in multiuser, multimedia networks.

Karin Klabunde studied computer science and artificial intelligence at the University of Koblenz, Germany and University of Edinburgh, Scotland. She joined Philips Research Laboratories in Aachen in 1990. She is involved in research on Intelligent Networks since 1991, especially in the areas of service creation and database technology. Currently, she is working on networked multimedia systems with special emphasis on session management for net-worked multi-user multimedia applications.

Ursula Konrads studied Computer Science at the Technical University of Aachen, Germany, where she graduated in 1985 and received her doctor's degree in 1989 in the field of formal languages. Since 1990 she has been with Philips Research Laboratories Aachen working on service architectures, especially service creation in Intelligent Networks and service control for multimedia applications. She is currently the project leader of Architecture for Interactive Networked Multimedia Applications.

Hermann Tjabben received the degree Diplom-Mathematiker in 1989 from the University Hannover, Germany. Since 1989 he has been with Philips Research Laboratories. He is currently a member of the department Telecommunication Architectures and Multimedia Systems. His current research focuses on protocol architectures for networked multi-user multimedia applications on top of ATM-LAN networks.

$\mathrm{He}$ is especially interested in employing formal description techniques such as SDL to improve the quality of communications software. 